\title{
Advanced Optical Delay Line Demonstrator
}

\author{
T. van den Dool*a, F. Kamphues ${ }^{\mathrm{a}}$, B. Fouss ${ }^{\mathrm{b}}$, K. Henrioulle ${ }^{\mathrm{b}}$, H. Hogenhuis ${ }^{\mathrm{c}}$ \\ ${ }^{\mathrm{a}}$ TNO TPD, Stieltjesweg 1, $2628 \mathrm{CK}$, Delft, THE NETHERLANDS \\ ${ }^{b}$ Micromega-Dynamics, Rue des Chasseurs Ardennais, B-4031, Angleur (Liège), BELGIUM \\ ${ }^{c}$ Dutch Space, Newtonweg 1, 2333 CP, Leiden, THE NETHERLANDS
}

\begin{abstract}
TNO TPD, in cooperation with Micromega-Dynamics and Dutch Space, has designed an advanced Optical Delay Line (ODL) for use in future ground based and space interferometry missions. The work is performed under NIVR contract in preparation for GENIE and DARWIN.

Using the ESO PRIMA DDL requirements as a baseline, the delay line can be used for PRIMA and GENIE without any modifications. The delay line design is modular and flexible, which makes scaling for other applications a relatively easy task.

The ODL has a single linear motor actuator for Optical Path Difference (OPD) control, driving a two-mirror cat's eye with SiC mirrors and CFRP structure. Magnetic bearings provide frictionless and wear free operation with zerohysteresis. The delay line is currently being assembled and will be subjected to a comprehensive test program in the second half of 2004.
\end{abstract}

Keywords: advanced optical delay line, ODL, GENIE, DARWIN, PRIMA, SiC mirrors, active magnetic bearings, nanopositioning, aperture synthesis

\section{INTRODUCTION}

TNO TPD and its partners have a broad experience with developing delay lines. Recent projects include the delay lines for the ASTRIUM Nulling Breadboard (under ESA contract), the ESO VLTI delay lines (developed by Dutch Space and TNO TPD) and a DARWIN cryogenic breadboard delay line (under ESA contract). Based on this experience, a delay line has been developed for use in the GENIE and PRIMA instruments. The long stroke actuator and guiding technology can also be applied in a future pre-DARWIN space mission.

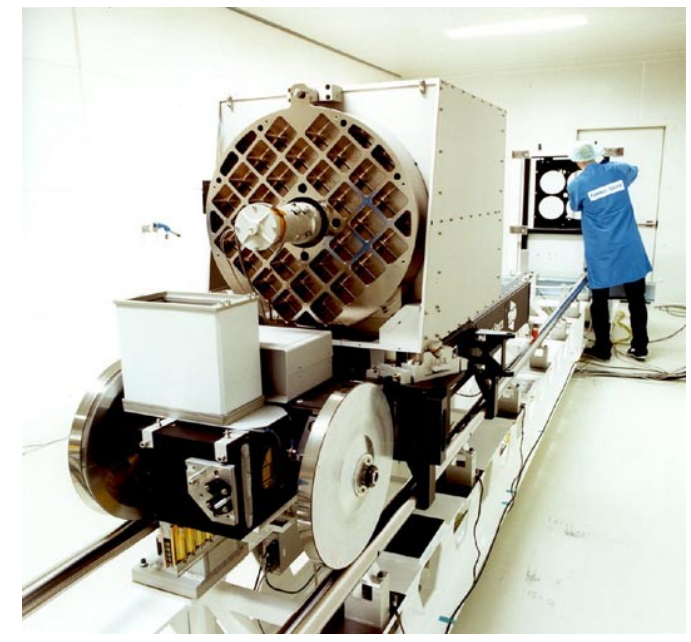

Fig. 1. ESO VLTI Delay Line (photo Dutch Space)

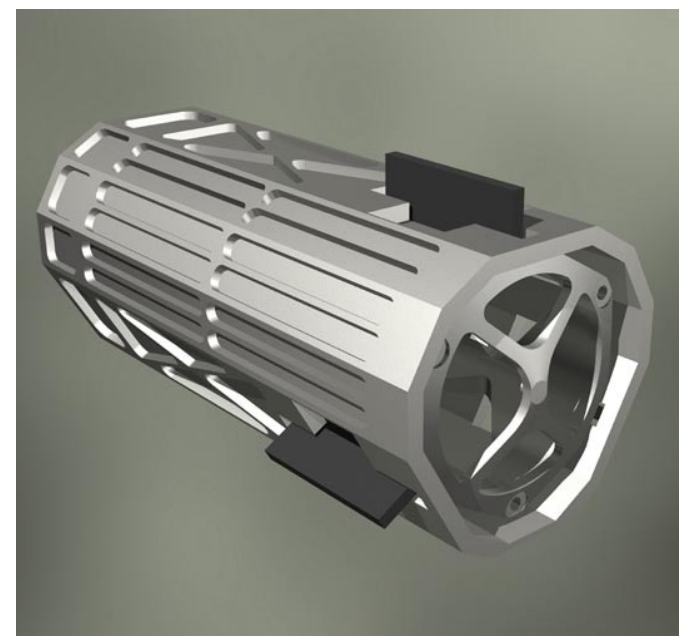

Fig. 2. ESA DARWIN breadboard cryogenic Delay Line

*dendool@tpd.tno.nl; phone 31-15-2692 457; fax 31-15-2692 111; www.tpd.tno.nl 
The main requirements for the Advanced Optical Delay Line Demonstrator have been derived from the ESO specifications for the PRIMA differential delay lines (DDL) [1], with an additional anticipated requirement for OPD stability for GENIE. An overview of the main requirements are given in table 1.

\begin{tabular}{|c|c|}
\hline Operational temperature & Ambient \\
\hline Pressure & Ambient and light vacuum (ca. 1 mbar) \\
\hline Temperature & Room temperature \\
\hline Mechanical environment & $\begin{array}{l}\text { The DDL shall meet their performance characteristic } \\
\text { requirements under micro-seismic activity as } \\
\text { specified in VLT-SPE-ESO-10000-0004. } \\
\text { They shall also sustain earthquakes with little or no } \\
\text { damage. A park mode is foreseen when a DDL does not } \\
\text { need to be active during observation. The retro- } \\
\text { reflector shall be put at a fixed, well-known and pre- } \\
\text { defined position outside of its active range. }\end{array}$ \\
\hline ODL stability & $\begin{array}{l}\text { PRIMA DDL: } \\
<8.0 \mathrm{~nm} \text { over any } 8 \mathrm{E}-3 \mathrm{~s} \text { in Visible spectral range } \\
<28.0 \mathrm{~nm} \text { over any } 39 \mathrm{E}-3 \mathrm{~s} \text { in Near IR spectral range } \\
<128 \mathrm{~nm} \text { over any } 335 \mathrm{E}-3 \mathrm{~s} \text { in Thermal IR spectral range } \\
\text { Anticipated for GENIE ODL: } \\
<5.0 \mathrm{~nm} \text { RMS }\end{array}$ \\
\hline OPD range (mechanical) & $70 \mathrm{~mm}$ \\
\hline $\begin{array}{l}\text { Slewing time } \\
\text { Slewing velocity }\end{array}$ & $\begin{array}{c}<5 \text { seconds } \\
>7 \mathrm{~mm} / \mathrm{s}\end{array}$ \\
\hline Fringe tracking and observation mode velocity & -500 to $+500 \mu \mathrm{m} / \mathrm{s}$ \\
\hline Dimensions (W x L x H) & $<240 \times 3000 \times 1800 \mathrm{~mm}$ \\
\hline Overall power dissipation & $<1 \mathrm{~kW}$ (for 8 delay lines) \\
\hline ODL power dissipation in interferometer lab & $<5 \mathrm{~W}$ (per delay line) \\
\hline F.o.V. & $15 \operatorname{arcmin}$ \\
\hline Pupil transfer & Yes \\
\hline Distance between input and output beam & $120 \mathrm{~mm}$ \\
\hline Optical beam diameter & $\begin{array}{l}\qquad>18 \mathrm{~mm} \\
\text { In combination with the pupil transfer and F.o.V. } \\
\text { requirements, this leads to a free aperture of } 55 \mathrm{~mm}\end{array}$ \\
\hline $\begin{array}{l}\text { Output beam lateral displacement } \\
\text { Output beam tilt } \\
\text { (both over the full actuation range) }\end{array}$ & $\begin{aligned} & <25 \mu \mathrm{m} \\
< & 1.5 \operatorname{arcsec}\end{aligned}$ \\
\hline Wavelength range & $0.42-28 \mu \mathrm{m}$ \\
\hline Wavefront distortion & $<25.0 \mathrm{~nm}$ RMS \\
\hline Transmission & $\begin{array}{l}\text { From } 0.42 \text { to } 1.0 \mu \mathrm{m}>80 \% \\
\text { From } 1.0 \text { to } 2.5 \mu \mathrm{m}>80 \% \\
\text { From } 2.5 \text { to } 28 \mu \mathrm{m}>90 \%\end{array}$ \\
\hline
\end{tabular}

Table 1. Advanced Optical Delay Line demonstrator main requirements 
A typical ODL system breakdown is given in figure 3.

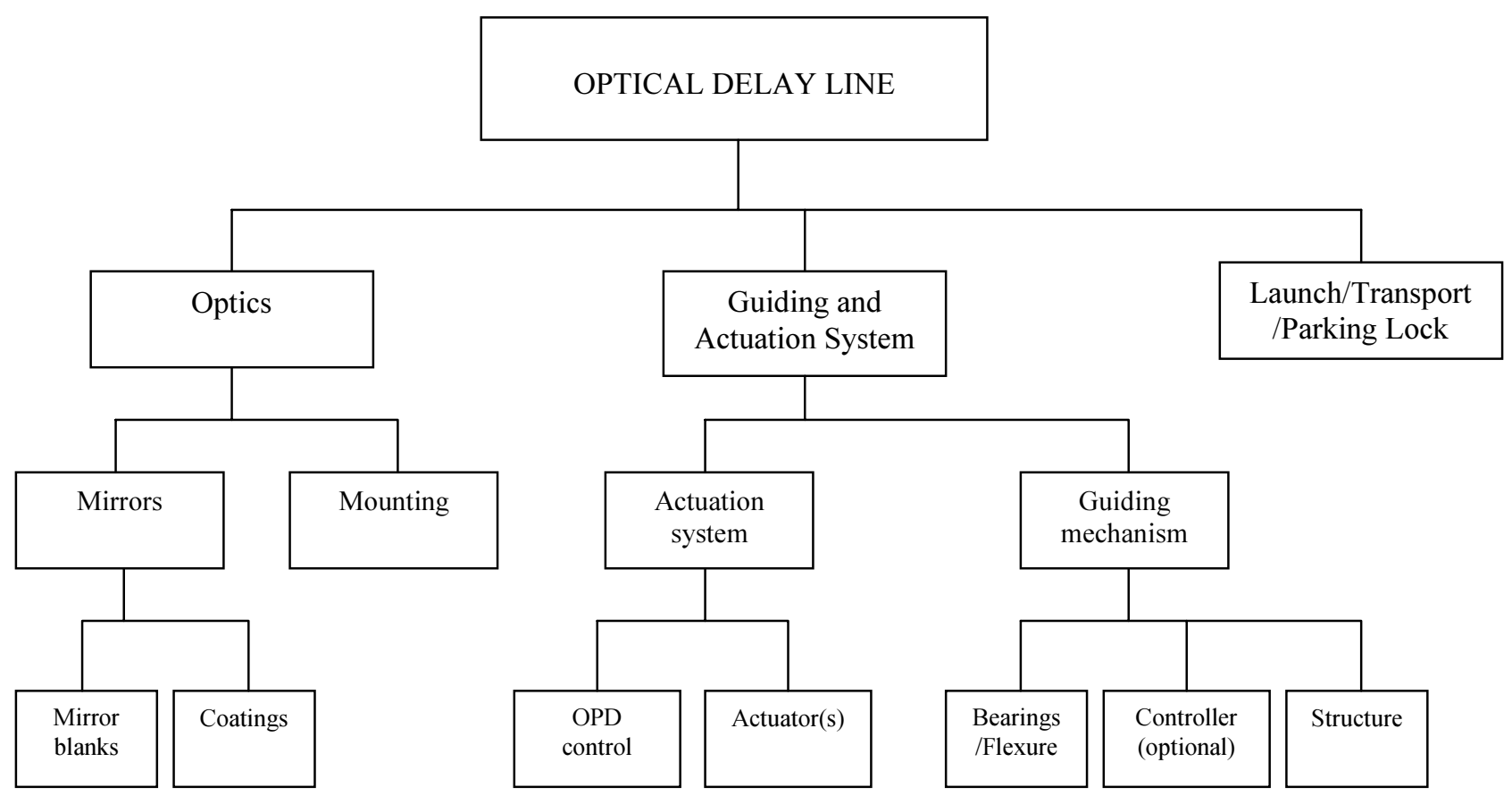

Fig. 3. Typical Optical Delay Line system breakdown

Within this study, the responsibilities for the design and development are divided as follows:

- TNO TPD - Project management, systems engineering, optical design and OPD control engineering

- Micromega-Dynamics - Guiding system development

- Dutch Space - Linear motor amplifier and controller electronics development

\section{CONCEPT SELECTION}

TNO TPD applies a minimum-number-of-stages philosophy to all delay lines it develops.

A smaller number of actuation stages simplifies the OPD control algorithm, reduces mechanical complexity and improves the optical quality of the outgoing beam.

For this particular application (GENIE and PRIMA), TNO TPD judged the possibility of a long stroke single stage delay line feasible, if the stringent targets for friction variations, hysteresis, linearity and moving mass can be met. 


\subsection{Optical layout}

The PRIMA DDL requirements for pupil transfer, input/output beam separation of $120 \mathrm{~mm}$ and $55 \mathrm{~mm}$ free aperture allow only for a cat's eye configuration.

Two cat's eye configurations were investigated:

- Two-mirror Cat's Eye, i.e. a combination of a focusing mirror and a planar mirror that is placed at the focus of that system.

- Three-mirror Cat's Eye, i.e. a combination of a focusing system (consisting of two mirrors) and a planar mirror that is placed at the focus of that system.

With the optical performance of both configurations almost identical, the preferred configuration is a two-mirror cat's eye, due to its higher transmission (only 3 reflections) and lower mechanical complexity (alignment).

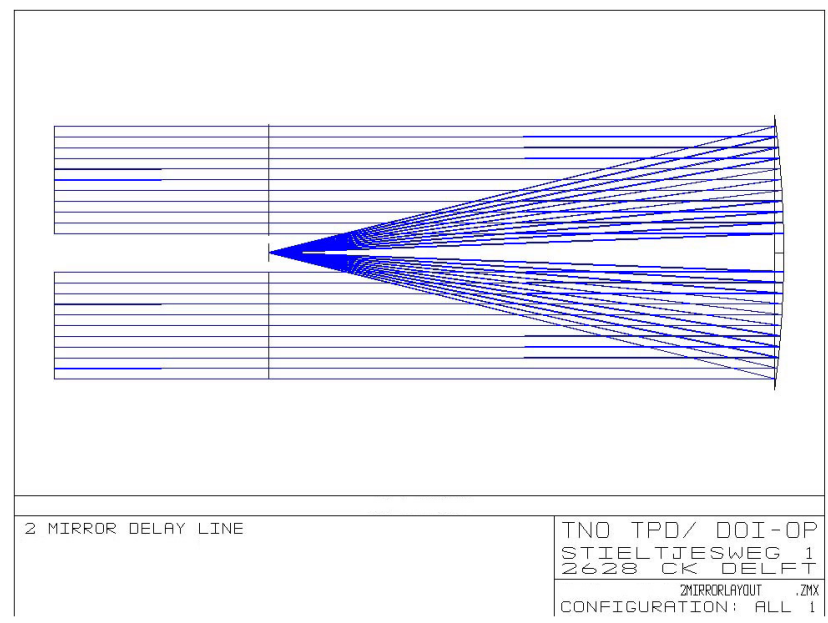

Fig. 4. Two-mirror Cat's Eye model

\subsection{Guiding system}

A number of options for the guiding system are available:

- ball or roller bearing

- air/hydraulic bearing

- flexure

- magnetic bearing

A ball bearing system shows considerable micro stick/slip and is sensitive to contamination.

These characteristics make it difficult to control the OPD variations with just a single actuator.

Air and hydraulic bearings are considered too complex for a vacuum application. With hydraulic bearings there is the additional concern of contamination.

While flexure provide smooth guiding, the required stroke of $70 \mathrm{~mm}$ makes them less attractive for this application (a double swing is large, heavy and complex). Flexure are also sensitive to misalignment errors (e.g. caused by small thermal variations).

For the required mechanical stroke of $70 \mathrm{~mm}$, a magnetic bearing based system offers the best performance in terms of moving mass, complexity, and guiding accuracy.

Due to the limited available width of $240 \mathrm{~mm}$ (and the minimum required mirror diameter of $200 \mathrm{~mm}$ ), the only place for the bearings is at the top or bottom of the cat's eye, requiring centralised digital control, due to the dynamics of this configuration. 


\subsection{Actuation and OPD control}

In a single stage delay line with high OPD stability $(<5 \mathrm{~nm})$ it is preferred to have the electrical connections on the static part. The following actuators were considered for OPD control:

- $\quad$ Surface Acoustic Wave (SAW)/Ultrasonic/Piezo motors

- Voice Coil

- Linear Motor

The performance of the first group of actuators (piezo material based) looks very promising, but the current maturity in linear drives is low. The issues of glitches, wear and noise cannot be properly assessed at this stage.

There is very little difference between the performance of a voice coil and a linear motor. For the given mechanical stroke of $70 \mathrm{~mm}$, a linear motor has a higher force to mass ratio than a voice coil, and is therefore the preferred solution.

The ODL shall be able to operate in three modes:

- Fringe acquisition ( $\mathrm{mm}$ range): to scan over the whole ODL range in order to find the coherence length range where fringes appear (interferences).

- Zero path difference locking ( $\mu \mathrm{m}$ range): to scan within the fringe range (coherence length of the light source) in order to find the zero path difference, where a constructive interference occurs (destructive in case an achromatic $\pi$-phase shifter has been introduced in one of the arms of the interferometer) for all wavelengths.

- Path stabilization and tracking (nm range): to compensate the OPD variations due to optical path disturbances (both mechanical and air turbulence) during the entire observation time (several hours).

The OPD control can be implemented in two ways:

- Analogue

- Digital
○ Fixed
- Re-programmable or adaptive

An assessment of the performance of the different type of OPD controllers shows:

- For high performance, the controller is often a complex transfer function, which is difficult to implement accurately in analogue electronics. This especially holds for higher frequencies where the plant (actuator to sensor transfer function) is complex.

- The disturbances may also change over time, for instance due to a change in conditions (e.g. temperature changes), and only an adaptive, or re-programmable controller would be able to cope with this.

A re-programmable or adaptive digital controller is the preferred solution for the GENIE and PRIMA environment.

\section{Summary}

The selected concept for the advanced ODL consists of:

Optics: 2-mirror cat's eye

Guiding: $\quad$ Magnetic bearings constraining 5 DOF with eddy current sensors and central digital controller

Actuation: $\quad$ Single stage with linear motor actuator and digital OPD controller. 


\section{DESIGN OF THE OPTICAL DELAY LINE}

\subsection{Retro-reflector}

The retro-reflector consists of a two-mirror cat's eye, containing one parabolic and one flat mirror. The parabolic M1 has a diameter of $200 \mathrm{~mm}$ (ESO I/F requirement) with a focal length of $700 \mathrm{~mm}$. The flat M2 has a diameter of $25 \mathrm{~mm}$.

The cat's eye has two openings for the science beam and two for a laser metrology beam.

The optical configuration is shown in figure 5.

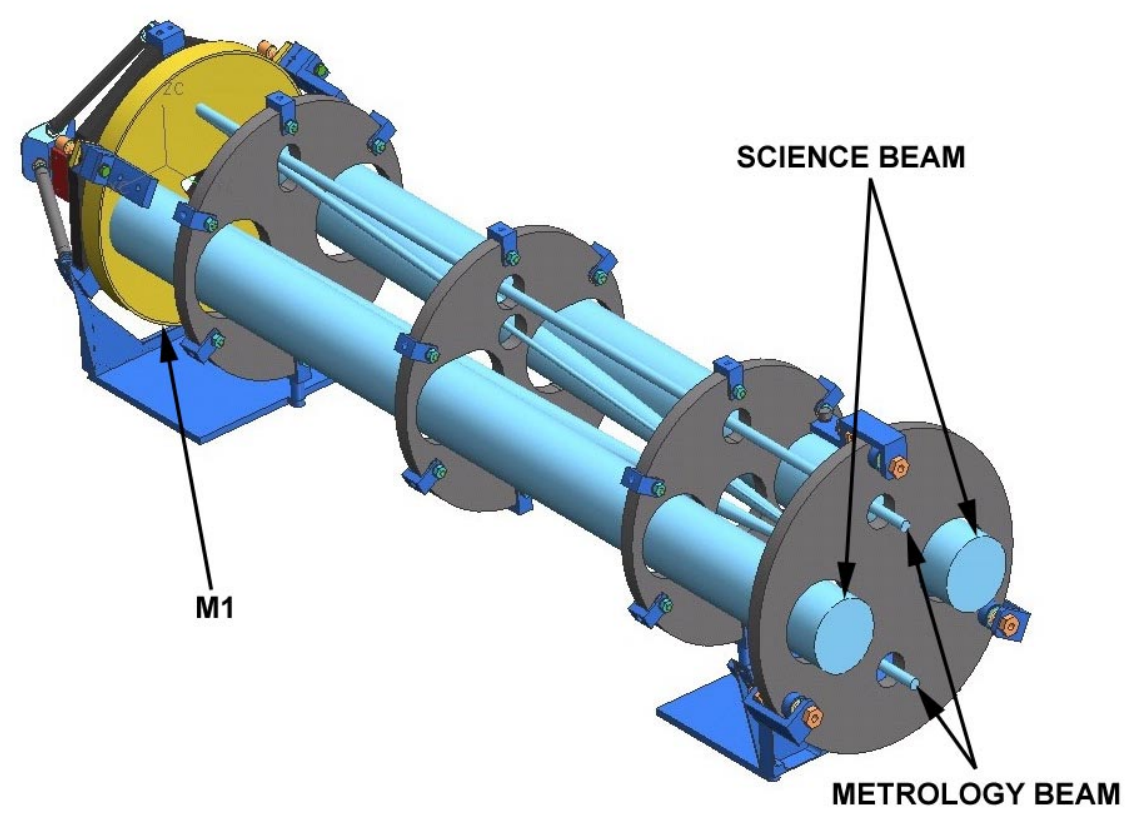

Fig. 5. Cat's Eye optical configuration

\section{Structure}

The use of magnetic bearings requires a high structural stiffness of the cat's eye, to avoid control flexibility interactions and subsequent bearing instability.

This calls for a lightweight retro-reflector design, using advanced materials such as CFRP and SiC.

The cat's eye consists of a tubular structure with internal baffles to suppress local modes.

The first natural frequency of the cat's eye (including mirrors) is $1082 \mathrm{~Hz}$. The first mode is shown in figure 6 .

The tube is made from high modulus CFRP prepreg material and has a quasi isotropic layup. In order to avoid excessive moisture expansion, a cyanate resin is used instead of epoxy resin. The selected prepreg material is M55J/CE3, made by ATK-COI.

The internal baffles are made of sandwich panels with T300 face sheet and foam core.

The open sides of the sandwich panels are covered with a single layer of CFRP, to avoid contamination of the mirrors by trapped dust particles.

The entire CFRP structure, including the precision machining, is manufactured by Futura Composites in the Netherlands. 


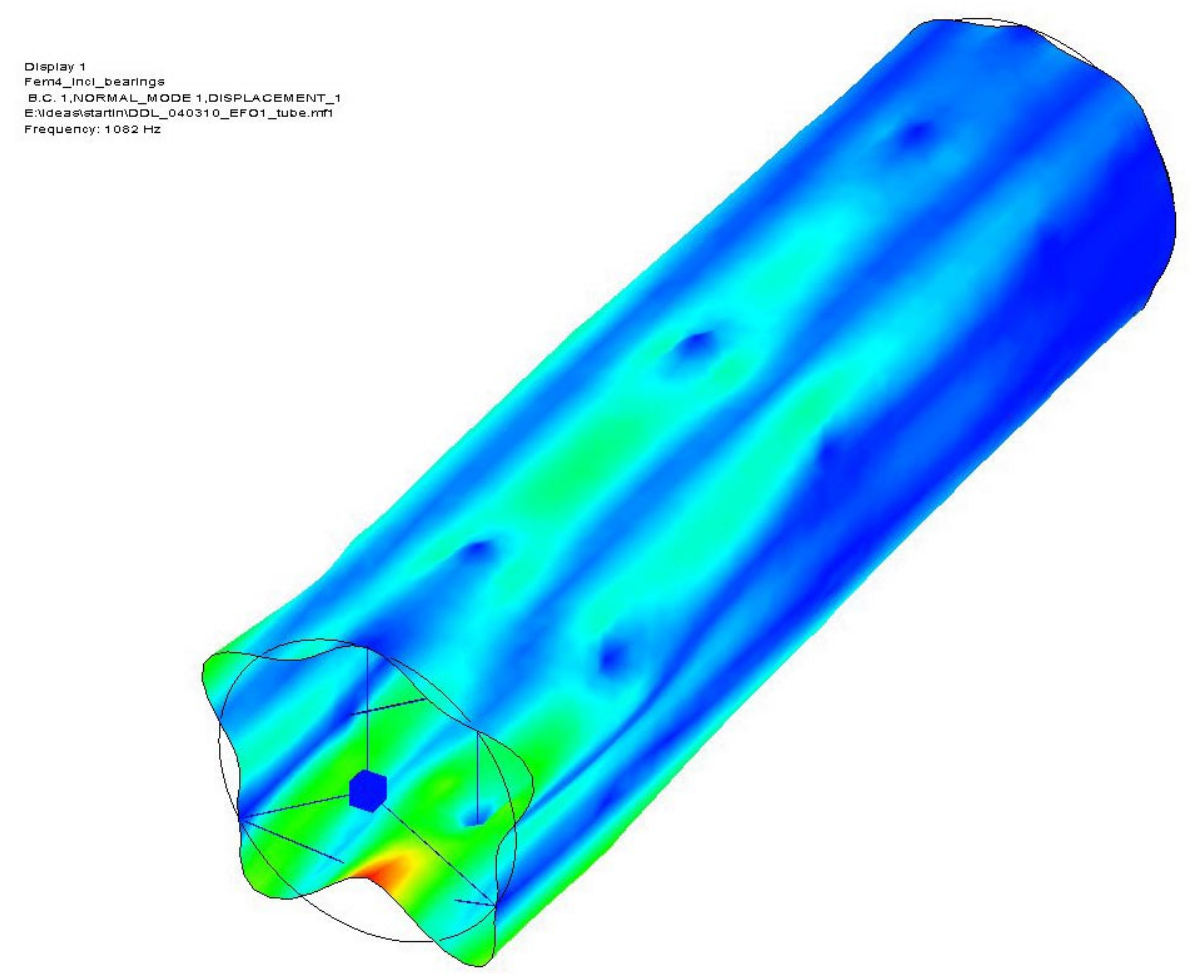

Fig. 6. Cat's eye first mode $(1082 \mathrm{~Hz})$

\section{Mirrors}

The CTE of a quasi isotropic M55J laminate is around $1 \mathrm{E}-6 \mathrm{~m} / \mathrm{m} / \mathrm{K}$ and requires mirrors with matching CTE for maximum (thermal) stability. Various suitable materials are available (Zerodur, ULE, SiC), but SiC offers the best performance in terms of specific stiffness.

The SuperSiC material, made by POCO Graphite in the USA, was selected for its high purity and relative ease of manufacturing. The mirror blanks are machined out of pure graphite and then converted to SiC. The Young's modulus is slightly lower than sintered SiC, but this can be offset by producing thinner wall thicknesses during machining.

The resulting lightweighted mirror has an areal density of approximately $15 \mathrm{~kg} / \mathrm{m}^{2}$.

The first natural frequency is around $2 \mathrm{kHz}$.

After $\mathrm{SiC}$ conversion, the mirrors are CVD coated and polished.

Polishing of both parabolic and flat mirror will be done in house by TNO TPD in cooperation with an external partner.

After polishing, a reflective Gold coating will applied to both mirrors to obtain the required transmission.

The parabolic M1 mirror is shown in figure 7.

The M1 is mounted in flexures to compensate for the small mismatch in CTE (around 1.3E-6 m/m/K) between the CFRP structure and the mirrors. For similar reasons, a set of metal adjustment washers are added between M2 and the structure to compensate for variations in focal length. 


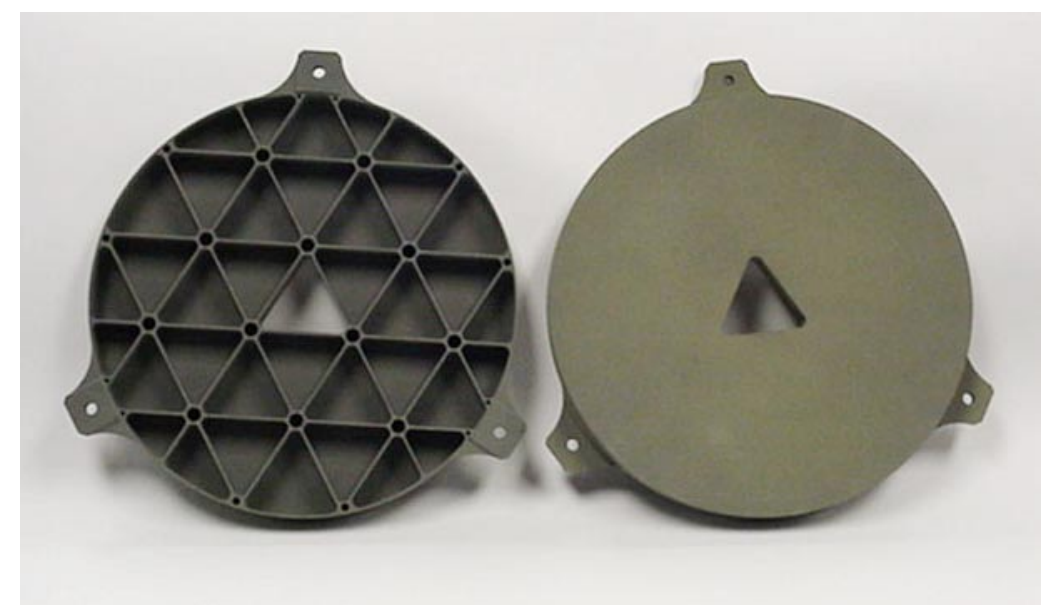

Fig. 7. ODL $200 \mathrm{~mm}$ diameter SiC M1 mirrors (photo POCO)

\subsection{Guiding Mechanism}

The guiding mechanism is based on active magnetic bearings. The magnetic bearing system has been designed by Micromega-Dynamics in Belgium, who have extensive experience with magnetic bearings, including application in space mechanisms such as MABE [12] and the DARWIN breadboard cryogenic delay line.

Magnetic bearings are relatively simple, and being contactless, do not require lubrication and do not wear. The inherent cleanliness makes them highly suitable for sensitive optical instruments.

In order to limit the power dissipation in the ODL, permanent magnet based reluctance force actuators will be used for the bearings. As the delay line requires operation in a $1 \mathrm{~g}$ environment, the permanent magnets carry around $95 \%$ of the mass of the moving part, to minimise power dissipation. The remaining mass (ca. 120 gram) is levitated by electromagnets.

Figure 8 shows the magnetic bearing configuration. A (moving) piece of soft iron is attracted by a permanent magnet. The system is inherently unstable. The permanent magnet is located in the middle of two soft iron parts. The permanent magnet produces a constant magnetic flux and the coils generate a variable magnetic flux that is added (or subtracted) to (from) the constant one. Eddy current sensors provide position information to the bearing controller, which in turn, keeps the moving soft iron part in its centre position.

Micromega has made a mathematical model of the ODL to optimise bearing layout and control bandwidth and calculate self induced perturbations.

The advanced ODL has five magnetic bearings to constrain five degrees of freedom (the OPD controller constrains the other degree of freedom). The centralised magnetic bearing controller will run on the same PC as the OPD controller. The bearings are located under the cat's eye, as shown in figure 9. 


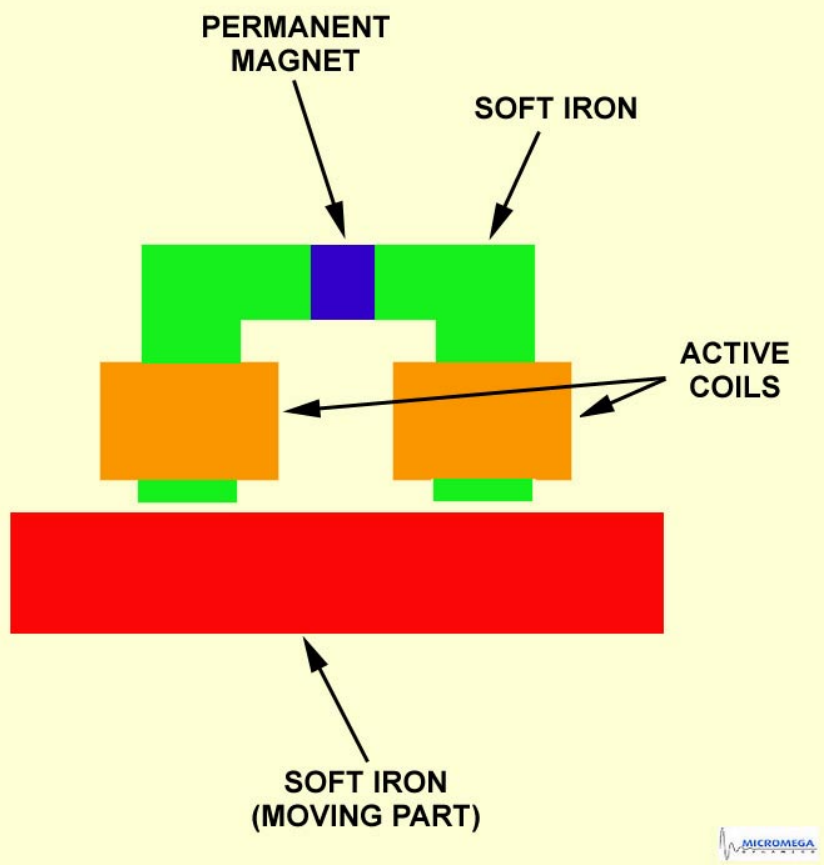

Fig. 8. Active magnetic bearing

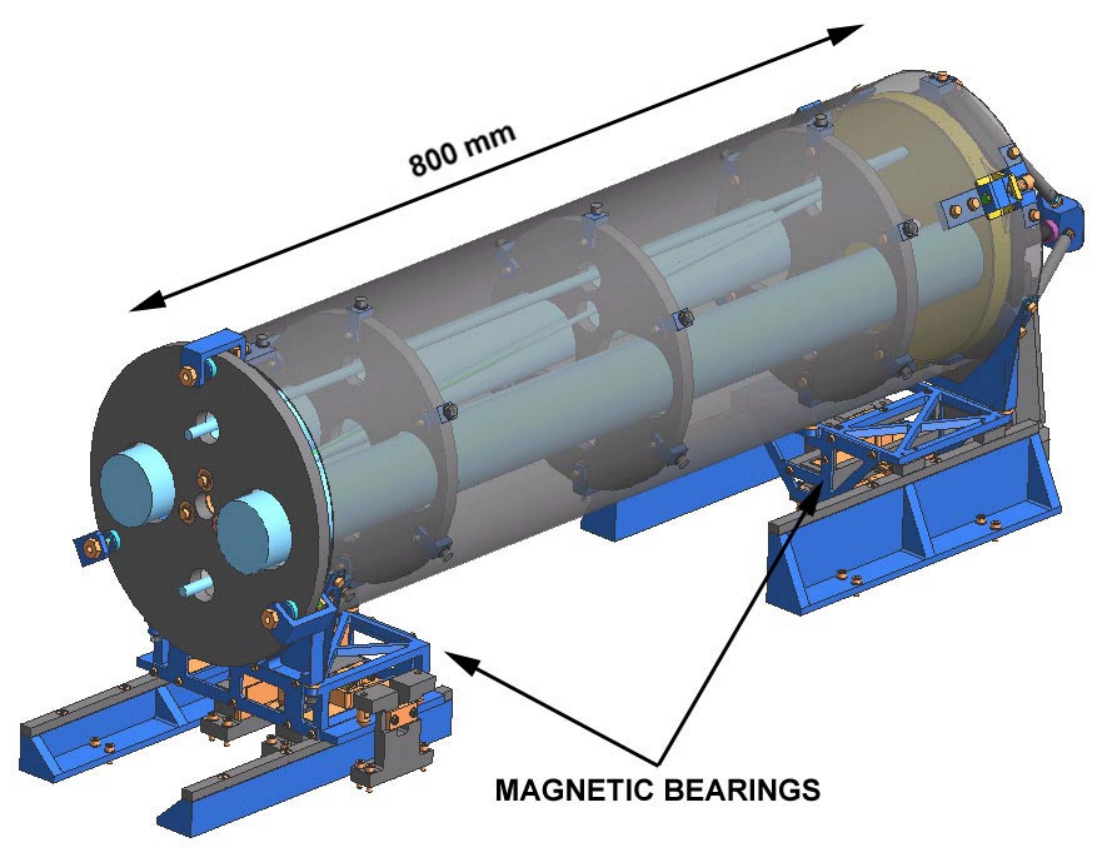

Fig. 9. Location of the magnetic bearings 


\subsection{Actuation and OPD Control}

\section{Actuator}

The cat's eye is actuated by a miniature linear motor. The development of the motor controller electronics is done in close cooperation with Dutch Space, and is based on the experience gained by Dutch Space with the VLTI delay lines.

The linear motor voice coil actuator is located at the back of the cat's eye and acts in the C.o.G. of the delay line.

The coil assembly is attached to the static part of the ODL and the magnet rod is attached to the moving part, thus preventing disturbance forces caused by electrical wires. The magnet rod is attached to the cat's eye structure with a CFRP truss, as shown in figure 10.

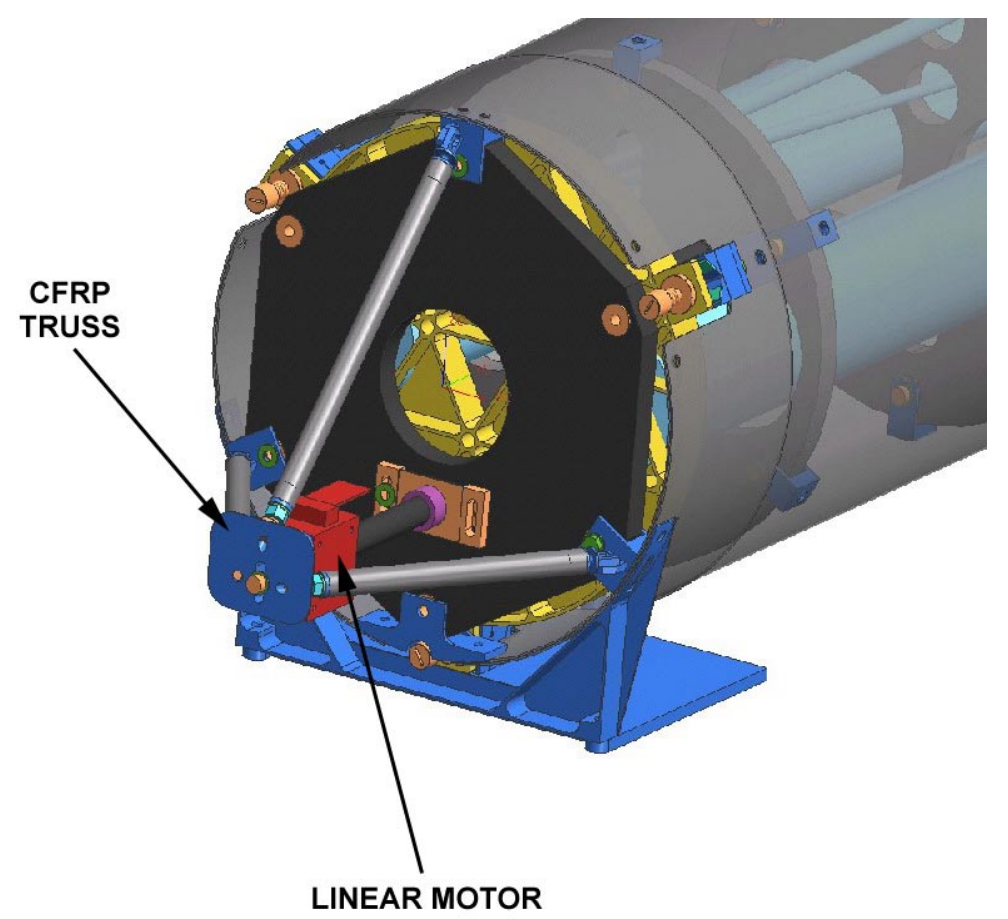

Fig. 10. Miniature Linear Motor

\section{OPD controller}

The advanced ODL will use a digital controller. The controller will be implemented on a real-time Linux PC to enable quick adjustment and fine tuning of control parameters during the development phase.

In path stabilization mode the control system needs to reject a stochastic-type disturbance by means of a feedback action (there is no advance information available). For this type of regulator system fundamental limits on the performance apply. The maximum, theoretical achievable disturbance rejection depends on the spectral characteristics of the disturbance together with the total loop delay. Here, we have assumed that the plant dynamics - other than the delay can be compensated for perfectly. In general, the maximum achievable disturbance rejection improves with a more narrowband disturbance spectrum and a smaller loop delay.

Control simulations have been performed to predict the controller performance with various loop delays and OPD sensor sampling rates. 
Experimental results at TNO TPD and Dutch Space on other delay lines, show that the predicted rejection ratio is achievable $[3,8,10]$. For the GENIE and PRIMA delay lines an attenuation of the disturbance of $>70 \mathrm{~dB}$ appears to be feasible (see figure 11), enabling better than $5 \mathrm{~nm}$ RMS OPD stability.

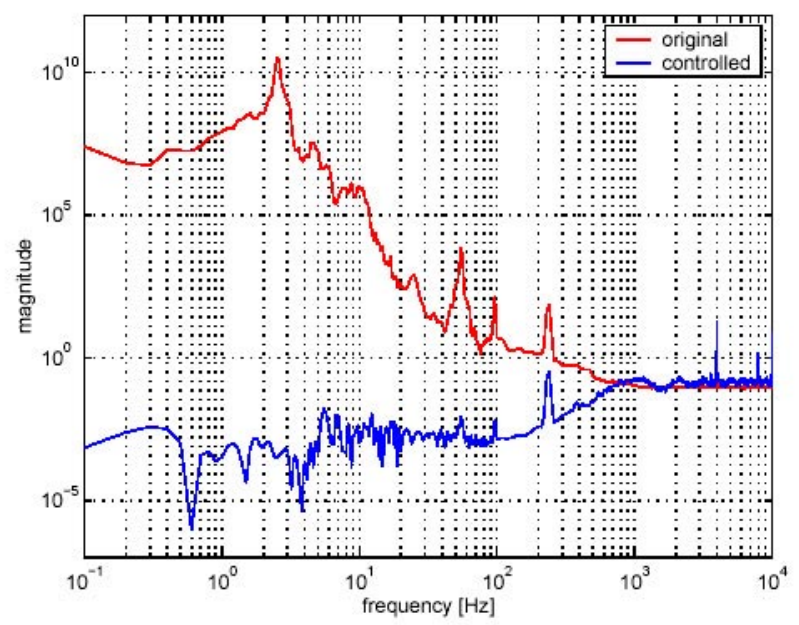

Fig. 11 Simulated disturbance rejection with a loop delay of $50 \mu$ s.

\section{CONCLUSION}

Analyses show that the design of an advanced single stage ODL can meet the requirements for GENIE and PRIMA.

The advanced ODL is currently being assembled and a comprehensive test program will start in June 2004, to demonstrate the critical parameters.

\section{AKNOWLEDGEMENTS}

TNO TPD would like to thank Joost Carpay (NIVR), Vincent Vlasblom (Futura Composites), Ed Lettner (ATK-COI) and Elmer Pennewaard (POCO Graphite) for their support during the development of the advanced delay line. 


\section{REFERENCES}

[1] Prima Differential Delay Line (DDL) Performance \& Technical Requirement Specifications, ESO VLT-SPE-ESO15720-2209 (http://www.mpia-hd.mpg.de/PRIMA-DDL/)

[2] B. Snijders et al., "Free-beam delay line for a multi-aperture optical space interferometer stabilized on a guide star", SPIE Vol. 2209, 1994

[3] B.C.Braam et al., "Kinematic six-ball guide for long stroke optical delay line", Proc. Sixth European Space Mechanisms \& Tribology Symposium, Zurich (Switzerland), 1995, ESA SP-374, August 1995

[4] R.J. Calvet et al., 'Enabling design concepts for a flight-qualifiable optical delay line', SPIE Vol. 3350 (1998), pp. 35-47.

[5] S. Mekid and M. Bonis, Conceptual design and study of high precision translational stages: application to an optical delay line, Precision Engineering (1997), Vol. 21, No. 1, p. 29 - 42.

[6] 'Optical Delay Line Nanometre Level Pathlength Control Law Design For Space-Based Interferometry', Robert L. Grogan, Gary H. Blackwood, and Robert J. Calvet. SPIE, Kona, Hawaii, March 1998, pp.14-25

[7] Y. Honma et al., 'Development of the fine delay line in the Mitaka optical and infrared array (MIRA) project', SPIE Vol. 3350 (1998), pp. 192 - 201.

[8] 'Active Vibration Control for an Optical Delay Line', N.J. Doelman, T.C. van den Dool. Proc. of Active 2002, pp.887-898

[9] B. Koehler et al., 'Optical delay line for stellar interferometer', SPIE Vol. 1236 (1990), pp. 463 - 472.

[10] F. Derie et al., 'VLTI delay lines: design, development, performance results, and commissioning on site', SPIE Vol. $4006(2000)$.

[11] A. Molenaar, H.F. van Beek, and M.J.L. Sanders, " A New Linear Magnetic Bearing Configuration for High Accuracy Positioning", in Proceedings of MAG97: Magnetic Bearings, Magnetic Drives and Dry Gas Seals Conference \& Exhibition (P. Allaire, ed.), (Alexandria, Virginia), pp. 313-322, Technomic Publishing Company, Inc, July 1997

[12] MABE, Fine Precision Mechanism Based on Magnetic Bearing Technology, Micromega Dynamics - Executive Summary (ESA Contract No 13676/99/NL/PA) 